\title{
Interfacing AI with Social Sciences: the Call for a New Research Focus in HCI
}

\author{
Hamed S. Alavi and Denis Lalanne \\ Human-IST Institute, University of Fribourg, Switzerland \\ hamed.alavi@unifr.ch, denis.lalanne@unifr.ch
}

\begin{abstract}
We provide arguments for the necessity of broadening the engagement of $\mathrm{HCI}$ in translating knowledge created in social sciences to a major force that can drive $\mathrm{AI}$ and direct the ways in which it will impact various aspects of our world. We begin to sketch the outline of this engagement as a research agenda within HCI in reference to some of the definitional manifesto on the HCI's foundational role [4] as an action science [3]. Also, a part of our own research that scrutinizes some of the major AI projects [1-2] informs the presented arguments.
\end{abstract}

\section{Keywords}

Sociological Conception of Artificial Intelligence - Smart Agenda - Science and Technology Studies (STS)

\section{Introduction}

Similar to many other domains of research and design, the future of HCI is increasingly bound with the advances in Artificial Intelligence (AI). This is attributed to the opportunities it can create to enhance the processes

How to cite this book chapter:

Alavi, H.S. and Lalanne, D. 2020. Interfacing AI with Social Sciences: the Call for a New Research Focus in HCI. In: Loizides, F., Winckler, M., Chatterjee, U., Abdelnour-Nocera, J. and Parmaxi, A. (eds.) Human Computer Interaction and Emerging Technologies: Adjunct Proceedings from the INTERACT 2019 Workshops. Pp. 197-202. Cardiff: Cardiff University Press. DOI: https://doi.org/10.18573/ book3.z. License: CC-BY 4.0. 
and methods of research, but also to the challenging questions it raises such as "explainability", "agency", "trustworthiness", "ethics", and so forth.

However, the discussion that we would like to instigate in this paper takes a different standpoint and seeks to pronounce a new responsibility, a crucial and urgent one, that is embedded in the HCI's special scientific placement. Departing from a widely acknowledged observation that despite the substantial and multifaceted impact of AI on human life social sciences are far from being at a leading position, we argue that HCI can play a key role in rectifying this disconnect. The task, in a nutshell, is to bring the knowledge created in various areas of social science to the format and position that can effectively shape the development of non-human intelligent actors and inform the policies governing whether and how they should be adopted. The eventual objective is to ensure that AI as such will take us to a "better future" where human values and priorities are advanced, and prevent it from falling into the other paths that empirical findings and discourses already can forecast the ramifications. In the interest of clarity, we constrain the discussion of AI to what we refer to as "major AI projects", projects such as Smart City or Personalized Learning that are initially backed by certain technological possibilities and produced a wave of corporate investments and academic investigations. This is our primary interest in AI. Instead of discussing AI as a broad (and rather ambivalent) concept, the objective is to provide a platform in which major AI projects can be meticulously analyzed using the frameworks constructed in the relevant domains of social science.

We develop our argument is three steps: 1) we start by highlighting a set of cross-domain concerns stemming from the current mechanisms through which major AI projects have been conceptualized and advancing. Then we describe 2) why we think HCI holds the methodological capabilities to rectify those problems, and why it is only HCI that can do so. In the end, we 3) try to extend this discussion beyond abstract reflections and begin to draft a research agenda that can reify some of the described objectives. Furthermore, we support our arguments throughout this text with one specific example of Autonomous Vehicles within the framework of Smart Cities as one major AI project.

\section{The problem of tech-oriented market-led AI}

Until social sciences produce a "sociological conception of AI" [6], the most conceivable forecast for the proliferation of AI relies on the existing unrivaled trend, in which it is the market-led tech sector that determines what AI is, how it should be invested in, and in what shape and function will reach the societies. In this model, our cities, our homes, our everyday social interactions, our education system and many fundamental aspects of our world will be subject of changes that are founded within the tech industry and, at its best, steered by the insights confined within the scope of computer and data sciences. 
The problem of such a model is beyond the fact that data and computer sciences lack the necessary knowledge. One should, more importantly, see the principles embedded at the core of data science that are in contradiction with some of the human values. For example, the inherent desire for clustering or classification of data has shown that can engender division, discrimination, and segregation in social realms - the problem that has been already seen in the context of social media [12], but also the same has begun to manifest itself in the physical social environments $[7,11]$.

To our observation, across different domains, there is no shortage of critical scholarship that identifies the risks in the currently dominant formulation of AI projects. As an example, for the case of Smart City, beginning with technology and not with urban knowledge is rigorously criticized. Within the urbanism research community, Hollands describes his concerns for the obfuscation of the negative effects of IT on cities by it's business motivated promoters [9], Greenfield warns a return to the failed utopias of 20th century high modernism [15], Kitchen forecasts the rise of technocratic governance coming with the wave of smart city [10], Vanolo criticizes the current conceptions of smart city for the creation of discrimination against the "non-smart" citizens [13], Datta demonstrates, from the analysis of currently existing smart cities, the justification for regimes and processes of land dispossession [8], and Wiig criticizes the priorities given to the attraction of global business against local urgencies in dominant conception of smart cities [14].

The domain of urbanism and the case of Smart City is one example. To similar extents, one can observe critical discourses of the tech-oriented and market-led AI across many strands of social science.

\section{The perennial mission of $\mathrm{HCI}$}

"HCI manages innovation to ensure that human values and human priorities are advanced, and not diminished through new technology. This is what created HCI; this is what led HCI onto and then off the desktop; it will continue to lead HCI to new regions of technology-mediated human possibility" [4]. It has been widely recognised, as exemplified in the above statement, that one of the perennial roles of our community is to search for, develop and apply various means of assessing technological innovations and their societal impacts. What we would like to highlight in this contribution is the necessity to found a special focus within HCI that investigates a specific type of projects, namely the highly invested and deeply impactful projects that capitalise on the advance in data and computer science to create non-human intelligent actors and integrate them into social contexts that they both utilise and modify. Projects such as self-driving vehicles, which are often rushed to performance test and production before understanding the changes they bring with themselves in terms of urban individual and collective experiences. 
The distinguishing attributes of major AI projects - e.g. speed of proliferation and propagation - we believe, entail multidisciplinary analytical scholarship that is proactive in terms of guiding the trends and preventive of futures in which "human values and priorities" would be "compromised".

In order to understand whether or not $\mathrm{HCI}$ is capable of carrying out the task of interfacing social sciences with AI projects, one should consider the record of HCI research and design in embarking upon multidisciplinary endeavors. HCI has a successful history of intertwining with reflections and concepts in the various domain of social science, adopting and re-appropriating research methods from those domains, and creating situations of mutual learning. It also has shown that can speak the technical language of computer and data science and be visionary in the realm of technologies.

At the end, we would like to go one step further and claim that the onus falls uniquely on us, the HCI research and design practitioners. This is not only an interdisciplinary involvement; HCI should rather take the lead and commit itself with the responsibility of proactively checking AI agenda with social sciences and ensuring that AI takes us to a better world. This is justified by the intermediary placement of HCI between computing power and human values. The evolution of HCI methods, concepts, and reflections originally to interface computing systems with humans, has situated our domain in a unique position to interface computer science with humanities. This a unique position for HCI, which implies a unique responsibility. The other fields with similar placements, such as Digital Humanities (DL) and Science and Technology Studies (STS), define themselves within more confined scopes and consequently are limited in terms of methodological capacities needed for the described research focus. Loosely termed, DL applies digital tools to the study of humanity without concerning much with the impact of various technologies on humanity, and in STS the rise and adoption of technologies are examined as a social process, retaining the focus on the technology or science and rather than their effect on societies.

\section{A new HCI research agenda}

Without aspiring to a complete or a final picture, in this section, we would like to briefly mention some of the constituents of a research program that can be conducive to the mentioned objectives:

- Rigorous analytical discussions should be developed that scrutinise major AI projects. The analytical frameworks are borrowed from the relevant domain of social science corresponding to the project to be discussed, which provide the researchers with a list of agreed-upon subject matters in the specific context of the study. For example, a critical discussion of self-driving cars would be structured based on topics of interest when accounting for car mobility in urban design. Topics such as spatial justice, 
public health and well-being, sustainability, congestion, urban forms, and so forth. The immediate questions to be answered are whether the current formulation of the AI project would have a positive or negative impact on each of those topics. Moreover, the list of topics can be complemented by questions on the new interactive experiences that the AI project itself would impose to its users. For example, questions surrounding interaction between pedestrians and the self-driving vehicles and how to build up the sense of trust towards them.

- The outcome of such analysis may be in the form of proposals for modifications or amendments to the current formulation of the project or generate alternative narrations, new perspectives, and new schematisms for creativity and development. For example, in the context of smart mobility, one may see the value in redirecting the attention to how AI can enhance active mobility (walking and cycling). The same strand of multidisciplinary work in the first step continues here, this time in creating a new vision of what urban scientists see as enhancing human priorities and what HCI researchers may foresee as trustful intelligent mobile actors in the city and a realistic adoption scenario (that matches the constraints of the cities and avoids the mess and myth of technological pledges [5]).

- To be able to become an effective force that guides the evolution of AI projects, the resulting discourses should extend their reach beyond the borders of academic environments and interact with people and public policy-makers. Therefore, an essential part of the task is to translate the created visions and insights into the appropriate format that can offer the public opportunities for collecting alternative perceptions of, for example, what a smart city, smart mobility, and eventually smart mobile citizens can be.

These are some of the interrelated steps that together can support the engagement of different stockholder of AI projects to question technological innovations and to seriously contribute in shaping their future ideas that we believe should be scaffolded within the scope of HCI research and design related to the underpinning characteristics of our field as briefly sketched previously.

\section{Concluding remarks}

The discussion presented in this contribution is grounded in the fact that AI as such can lead our world to various futures. It can lead our cities to be even more car-dependent (through the promotion of autonomous vehicles); it can instead enhance active mobility (walking and cycling), make possible sustainable use of urban spaces and create human-scale public spaces. City is one example; such alternative futures extend to many aspects of life. The discourse that we initiate in this paper foregrounds the responsibility of HCI in studying such futures and providing directions for AI adoption policies. 


\section{References}

1. Alavi, Hamed S., et al. "Is Driverless Car Another Weiserian Mistake?" Proceedings of the 2017 ACM Conference Companion Publication on Designing Interactive Systems. ACM, 2017.

2. Alavi, Hamed, and Farzaneh Bahrami. "Walking in smart cities." Interactions. 26(2) (2019): 66-68.

3. Argyris, Chris, Robert Putnam, and Diana McLain Smith. Action science. Vol. 13. Jossey-Bass Inc Pub, 1985.

4. Carroll, John M., and Mary Beth Rosson. "Wild at home: The neighborhood as a living laboratory for HCI." ACM Transactions on Computer-Human Interaction (TOCHI), 20(3) (2013): 16.

5. Dourish, Paul, and Genevieve Bell. Divining a digital future: Mess and mythology in ubiquitous computing. Mit Press, 2011.

6. Mlynar, Jakub, et al. "Towards a Sociological Conception of Artificial Intelligence." International Conference on Artificial General Intelligence. Springer, Cham, 2018.

7. Soderstrom, Ola, Till Paasche, and Francisco Klauser. "Smart cities as corporate storytelling." City, 18(3) (2014): 307-320.

8. Datta, A., 2015. New urban utopias of postcolonial India: Entrepreneurial urbanizationin Dholera smart city, Gujarat. Dialogues in Human Geography, 5(1), pp. 3-22.

9. Hollands, R.G., 2008. Will the real smart city please stand up? Intelligent, progressive or entrepreneurial?. City, 12(3), pp. 303-320.

10. Kitchin, R., 2014. The real-time city? Big data and smart urbanism. GeoJournal, 79(1), pp. 1-14.

11. McFarlane, C., and Sderstrm, O., 2017. On alternative smart cities: From a technology-intensive to a knowledge-intensive smart urbanism. City, 21(3-4), pp. 312-328.

12. Qian, X., Feng, H., Zhao, G., and Mei, T., 2013. Personalized recommendation combining user interest and social circle. IEEE transactions on knowledge and data engineering, 26(7), pp. 1763-1777.

13. Vanolo, A., 2014. Smartmentality: The smart city as disciplinary strategy. Urban studies, 51(5), pp. 883-898.

14. Wiig, A., 2015. IBM's smart city as techno-utopian policy mobility. City, 19(2-3), pp. 258-273.

15. Greenfield, A., 2013. Against the Smart City: A Pamphlet. This is Part I of "The City is Here to Use". Do projects. 